INPLASY

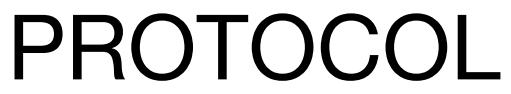

To cite: Dang et al. The effect of smoking status on efficacy of immune checkpoint inhibitors in metastatic nonsmall cell lung cancer: a systematic review and metaanalysis. Inplasy protocol 202140025. doi: 10.37766/inplasy2021.4.0025

Received: 05 April 2021

Published: 06 April 2021

Corresponding author: Jun Dang

dangjunsy@163.com

Author Affiliation:

The First Hospital of China Medical University

Support: None.

Review Stage at time of this submission: Data analysis.

Conflicts of interest:

None declared.

\section{The effect of smoking status on efficacy of immune checkpoint inhibitors in metastatic non-small cell lung cancer: a systematic review and meta-analysis}

Dang, J1; Dai, L2; Jin, B3; Liu, T4; Chen, J5.

Review question / Objective: It remains uncertain whether patients smoking status can effect efficacy of immune checkpoint inhibitors (ICls) in metastatic non-small cell lung cancer (NSCLC). We performed a meta-analysis to address this issue.

Condition being studied: To date, there is still no randomized controlled trial which has specifically assessed survival benefits from immune checkpoint inhibitors in smokers and never smokers with NSCLC respectively. Whether smoking status can act as a predictive marker of response to immune checkpoint inhibitors remains uncertain.

INPLASY registration number: This protocol was registered with the International Platform of Registered Systematic Review and Meta-Analysis Protocols (INPLASY) on 06 April 2021 and was last updated on 06 April 2021 (registration number INPLASY202140025).

\section{INTRODUCTION}

Review question / Objective: It remains uncertain whether patients smoking status can effect efficacy of immune checkpoint inhibitors (ICls) in metastatic non-small cell lung cancer (NSCLC). We performed a meta-analysis to address this issue.
Condition being studied: To date, there is still no randomized controlled trial which has specifically assessed survival benefits from immune checkpoint inhibitors in smokers and never smokers with NSCLC respectively. Whether smoking status can act as a predictive marker of response to 
immune checkpoint inhibitors remains uncertain.

\section{METHODS}

Participant or population: Patients with metastatic non-small cell lung cancer.

Intervention: Immune checkpoint inhibitors.

\section{Comparator: Chemotherapy.}

Study designs to be included: Literature search, inclusion and exclusion criteria, data extraction and quality assessment, statistical analysis, data analysis and interpretation, manuscript writing, and final approval of manuscript.

Eligibility criteria: (1) randomized controlled trials in advanced NSCLC; (2) compared immune checkpoint inhibitors (alone or in combination with other agents) with chemotherapy; (3) reported overall survival (OS) and/or progression-free survival (PFS) data in each arm, and data regarding smokers (defined as current and/or former smokers) and never smokers could be retrieved respectively; (4) published in English. If studies had multiple publications, the most recent one was used.

Main outcome(s): Effect of smoking status on immune checkpoint inhibitors efficacy in metastatic non-small cell lung cancer. OS and PFS are the primary outcomes of interest. HRs and their $95 \% \mathrm{Cls}$ are used as summary statistics.

Quality assessment / Risk of bias analysis: Risk of bias of individual trials is independently assessed by two authors, using the Cochrane Risk of Bias Tool. The studies are finally rated as low (all domains indicated as low risk), high (one or more domains indicated as high risk), and unclear risk of bias (more than three domains indicated as unclear risk).

Strategy of data synthesis: Literature search, data extraction and Quality assessment for eligible articles are performed by two authors independently.
Statistical analysis will be performed using the software Review Manager 5.3 (Cochrane Collaboration, Oxford, UK). OS and PFS are the primary outcomes of interest. The heterogeneity among studies is estimated by the Chi-square (2) and Isquare (12) test. Meta-regression analysis is conducted to search for the sources of heterogeneity. Sensitivity analysis is performed to verify the stability. Publication bias is evaluated by Begg's test, the Egger's test, and the funnel plot.

Subgroup analysis: Subgroup analyses are planed to perform according to treatment modality (montherapy/combination therapy), treatment line (first-line/ subsequent-line), and PD-L1 expression level $(<1 \% / \geq 1 \% / \geq 50 \%)$.

Sensitivity analysis: Sensitivity analysis was performed to verify the stability of the pooled results by removing the data of an individual study each time.

\section{Country(ies) involved: China.}

Keywords: immune checkpoint inhibitors; chemotherapy; non-small cell lung cancer; smoking status; meta-analysis.

Contributions of each author:

Author 1 - Jun Dang.

Author 2 - Linzheng Dai.

Author 3 - Bo Jin.

Author 4 - Tingting Liu.

Author 5 - Jun Chen. 